\title{
Adopción de tecnología de distribución y control del agua en las Comunidades de Regantes de la Región de Murcia
}

\author{
Francisco Alcón ${ }^{\mathrm{a}}, \mathrm{M}^{\mathrm{a}}{ }^{\mathrm{a}}$ Dolores de Miguel $^{\mathrm{a}}$, Michael Burton ${ }^{\mathrm{b}}$
}

\begin{abstract}
RESUMEN: En el presente trabajo se estudia el proceso de adopción de tecnología de distribución y control de agua que las Comunidades de Regantes de la Región de Murcia han llevado a cabo desde el año 1975 hasta 2005. Se analiza el tiempo que transcurre desde que se constituye la Comunidad de Regantes hasta que toma la decisión de adoptar utilizando Análisis de Duración. Se identifican como aceleradores del proceso de adopción la posesión de un pozo de apoyo, el empleo de un sistema tarifario variable en función del consumo, los efectos de las políticas de subvenciones a las obras de modernización y mejora de los regadíos y las sequías.
\end{abstract}

PALABRAS CLAVE: Análisis de duración, comunidad de regantes, función de riesgo, tecnología de riego.

Clasificación JEL: Q16, Q25, D71.

\section{Adoption of water conveyance and monitoring technology in the irrigation communities in the Region of Murcia (Spain)}

SUMMARY: The present paper analyses the process of adopting water management and control technology in the irrigation communities in the region of Murcia from 1975 to 2005. Duration Analysis has been used to combine the technological process with the time taken from the initial stages of the irrigation community until the execution of this plan. The ownership of a subterranean water well and the use of a variable tariff system according to the water consumed have been identified as the moving forces of the adoption process. The policies of subsidies toimprove irrigation technology areas and drought have also affected the adoption decisions.

KEYWORDS: Duration analysis, irrigation community, hazard function, irrigation technology.

JEL classification: Q16, Q25, D71.

a Departamento de Economía de la Empresa. Universidad Politécnica de Cartagena.

b School of Agricultural \& Resource Economics. University of Western Australia.

Agradecimientos: El presente trabajo forma parte de las investigaciones realizadas dentro del Proyecto I+D+I de referencia AGL 2002-04251-C03-01, financiado por el Ministerio de Ciencia y Tecnología y los Fondos FEDER. Los autores agradecen los comentarios de los revisores anónimos y del ponente.

Dirigir correspondencia a: Francisco Alcón.E-mail: francisco.alcon@upct.es

Recibido en enero de 2007. Aceptado en marzo de 2008. 


\section{Introducción}

La marcada variabilidad hidrológica interanual de la Cuenca del Segura, la apertura de los mercados europeos a los productos españoles y la intensificación de la agricultura murciana son tres elementos que han propiciado un importante desarrollo tecnológico en el sector agrario. Entre las tecnologías implantadas cabe destacar el empleo de tecnología de riego que mejora la gestión y reduce el uso del agua. Esto ha generado un importante ahorro económico, dado que el precio del agua en la Región de Murcia es de los más elevados de España ${ }^{1}$.

El cambio tecnológico implica dos procesos consecutivos, la generación de una nueva tecnología y su adopción por los usuarios, ya que el beneficio de esta tecnología solo revertirá en la sociedad una vez que haya sido adoptada. De ahí que la difusión de una innovación a través de la población de potenciales adoptantes es uno de los principales determinantes del crecimiento económico (Stoneman, 1986).

En la actualidad se están desarrollando políticas que fomentan la adopción de tecnologías de riego y potencian la conservación de los recursos naturales. La Directiva Marco de Aguas de la Unión Europea² (DMA), aprobada en 2000, reconoce que el agua no es un bien comercial y establece un marco comunitario para la protección de las aguas y del medio ambiente (MMA, 2007).

Al amparo del Texto Refundido de la Ley de Aguas $^{3}$, el Plan Nacional de Regadíos - Horizonte $2008^{4}$ (PNR) estableció una reorientación en la política nacional de regadíos que considera la modernización de infraestructuras de distribución y control del agua de riego, como una de las soluciones al problema del regadío. Con ello se pretende racionalizar el uso de los recursos, reducir la contaminación de origen agrario y promover innovaciones en los sistemas de riego para disminuir los consumos de agua (MAPA, 2001).

La adopción de sistemas de distribución y control del agua, que corresponde a las Comunidades de Regantes (CC.RR.), se ha venido realizando de forma general, a través de los planes de modernización, mejora y consolidación de regadíos. Estos planes, considerados un determinante clave para mejorar el uso de los escasos recursos hídricos (Cason y Uhlaner, 1991), consisten en la ejecución de las obras y actuaciones necesarias para incrementar la eficiencia técnica del uso del agua, reducir los impactos ambientales del regadío, ahorrar agua y mejorar la productividad económica de los regadíos (MAPA, 2001).

La adopción es un proceso basado en una secuencia de decisiones que los individuos toman para decidir si adoptan o rechazan una innovación (Gatignon y Robertson, 1991). Una vez adoptada la tecnología, los beneficios potenciales que conlleva recaerán sobre los adoptantes, contribuyendo globalmente a la mejora del bienestar social (Lindner, 1987; Rogers, 2003).

\footnotetext{
${ }^{1}$ Según MMA (2007) el precio del agua en la Cuenca del Segura es de $462 € /$ Ha e incluso se han llegado a estimar costes de extracción hasta $0,79 € / \mathrm{m}^{3}$.

2 DIRECTIVA 2000/60/CE, de 23 de octubre.

3 R.D. $1 / 2001$, de 20 de julio.

${ }^{4}$ R.D. 329/2002, de 5 de abril.
} 
Partiendo de que la adopción es rentable, la tecnología de riego se ha difundido lentamente. Los motivos que han originado esta escasa velocidad de difusión han sido analizados por diferentes autores. Schaible et al. (1991) encontraron que la escasa velocidad, en ausencia de cambios institucionales, era debida a que los agricultores no percibían la escasez. Por otro lado, Zilberman et al. (1995) establecieron que los eventos extremos, como las sequías, incrementaban la velocidad de difusión de tecnología de riego. Esto se encuentra acorde con la hipótesis de escasez de recursos planteada por Hayami y Ruttan (1985) y corroborada por Foltz (2003), bajo la cual, el ritmo de adopción es establecido por la escasez de recursos y el precio sombra de éstos. Por otro lado, Carey y Zilberman (2002) exponen que el establecimiento de una garantía de suministro ofrecida a través de fuentes alternativas, como los mercados de agua, reducirá la velocidad de difusión. Es decir, mientras que unos trabajos mantienen la idea de que la reducción de los recursos naturales y el incremento de su precio aumentan las tasas de adopción, otros se basan en que la incertidumbre relacionada con la disponibilidad del agua promueve las decisiones finales de adoptar, y por lo tanto, la velocidad de difusión.

Los factores que afectan a la velocidad del proceso de adopción de tecnología se pueden clasificar en cinco grupos: características del agricultor, factores económicos, características de la explotación, características de la innovación y factores del entorno. Aunque son muchos los factores que componen cada grupo, se resaltarán los de mayor trascendencia en las tecnologías de riego. Entre las características del agricultor cabe destacar el nivel de formación (Sidibe, 2005), el grado de aversión al riesgo (Feder, 1980; Marra et al., 2003), las percepciones de la tecnología (Adesina y Zinnah, 1993; Adesina y Baiduforson, 1995) y el poder de persuasión de los distintos canales de comunicación (Alcón et al., 2006). Dentro de los factores económicos resaltan el tamaño empresarial (Negri y Brooks, 1990; Green et al., 1996) y la disponibilidad de capital (Smale et al., 1994; Foltz, 2003). Las características de la explotación suelen estar marcadas por factores agronómicos (Green et al., 1996; Green y Sunding, 1997), las de la innovación por la ventaja relativa que genera la incorporación de la innovación (Schaible et al., 1991) y, los factores del entorno recogen los aspectos sociales y políticos de la zona en la que se desarrolla la actividad, generalmente basados en políticas tarifarias (Caswell y Zilberman, 1985; Caswell et al., 1990; Negri y Brooks, 1990) y de ayudas a la innovación tecnológica (Dinar y Yaron, 1992).

Estos factores, que tienen influencia sobre la decisión de los agricultores individuales frente a la adopción de tecnologías de riego, también pueden aplicarse a las CC.RR., dado que las opiniones conjuntas de los comuneros determinan la toma de decisiones en la asamblea de la CR, y por lo tanto, la disposición de la CR a la hora de abordar un plan de modernización (Sumpsi et al., 1998). Por ello, las características más importantes que definen las CC.RR. españolas, identificadas por Sumpsi et al. (1998), han sido englobadas dentro de estos cinco grupos de factores. El sistema de distribución y control del riego y el sistema tarifario establecido estarían comprendidos dentro de las características de la CR, considerada ésta como unidad social en la que cada individuo participa en su gestión. El precio del agua sería un factor económico. El origen del agua pertenecería al grupo de factores que definen las caracte- 
rísticas de las explotaciones que conforman el perímetro regable. La ventaja relativa del uso de esta tecnología (p.e., la posibilidad de realizar mediciones de consumos precisas) se englobaría dentro de las características de la innovación, y la disponibilidad del agua o la existencia de ayudas gubernamentales serían factores del entorno de las CC.RR.

Las CC.RR. desempeñan un papel muy importante en la sociedad rural, ya que son las encargadas de adminstrar el agua y distribuirla entre sus usuarios. Las actuaciones de las CC.RR. han sido reflejo de las estructuras sociales, económicas y políticas, han asignado los usos del agua y han contribuido a la historia hidrológica atribuida a estas estructuras (Wulfhorst, 2002). En la Región de Murcia ya aparecían las CC.RR. como asociaciones que, con diferentes denominaciones, poseían un sistema de organización mediante el cual administraban y distribuían el agua destinada al regadío de los cultivos. Estas CC.RR. realizaban una distribución del agua en común utilizando acequias de riego, incluso antes de la conquista árabe del siglo VIII, poniendo de manifiesto la conveniencia de la gestión del agua y su repercusión en las necesidades de la misma (Nadal, 1980; Box, 1992). A pesar de ello, no se ha encontrado en la literatura científica de adopción estudios empíricos donde la unidad de análisis sea la CR como centro decisor.

Por lo tanto, para estudiar el fenómeno de adopción de tecnología se encuentran en la literatura dos perspectivas o niveles (Feder y Umali, 1993). Un nivel micro, donde cada unidad decisional analizada debe decidir si adopta o no una innovación, existiendo una serie de factores que afectan a esta decisión. Y un nivel macro, donde el patrón de adopción de todas las unidades decisionales es examinado en el tiempo para identificar la tendencia específica dentro del ciclo de difusión, partiendo de un momento determinado donde la innovación está ya en uso y sin considerar el proceso de innovación.

La metodología de Análisis de Duración (AD) permitirá abordar los dos niveles de forma simultánea, dado que la dicotomía existente entre la difusión como un proceso (nivel macro) y la adopción debida a la heterogeneidad individual (nivel micro) es un artificio en el que la curva de difusión es un agregado de las decisiones de adopción individuales.

En este contexto, el presente trabajo tiene como objetivo describir el proceso de adopción de tecnología de distribución y control del agua entre las CC.RR. de la región de Murcia, identificando los factores asociados con la decisión de adoptar y estableciendo la importancia relativa de los mismos sobre esta decisión, utilizando la metodología de AD. Este proceso de adopción tecnológica puede servir de apoyo al establecimiento de políticas de modernización de regadíos que actualmente se están llevando a cabo tanto en España como en Europa.

\section{Metodología}

Entre los modelos temporales de adopción, el AD permite estudiar la adopción a nivel individual y considerar con posterioridad la difusión tecnológica. Con respecto a los modelos tradicionales (logit, probit, etc.) el AD presenta la ventaja de explicar, 
no solamente qué individuos adoptan, sino también cuanto tiempo han tardado en realizarlo, ofreciendo la posibilidad de explorar especificaciones alternativas para la curva de difusión en forma de $S$ (Abdulai y Huffman, 2005).

Son escasos los estudios con esta técnica en el campo de las ciencias agrarias. Entre ellos, Burton et al. (2003) analizaron la adopción de técnicas de producción orgánica en el Reino Unido; Souza Filho et al. (1999) identificaron los factores que influenciaban la adopción de agricultura sostenible en Brasil; Fuglie y Kascak (2001) la aplicaron a tecnologías diseñadas para reducir las externalidades ambientales de la agricultura en una amplia zona del oeste de EE.UU.; y D'Emden et al. (2006) analizaron la adopción de prácticas de conservación del suelo en Australia.

Con el propósito de describir el proceso de adopción e identificar estadísticamente aquellos factores que presentan un efecto significativo sobre la longitud del tiempo de adopción, el AD estudia la diferencia de tiempo $T$ existente entre dos estados alternativos y excluyentes por los que pasa un individuo desde que es un potencial adoptante hasta que adopta. Esto es, el tiempo que transcurre desde que la CR se constituyó hasta que decide ejecutar un plan de modernización de su infraestructura de distribución y control del agua. El rango de tiempo comienza en el año 1975, cuando aparece la tecnología de riego en el mercado o el año en que se constituye la $\mathrm{CR}$, si es que éste es posterior, y finaliza el primer año de ejecución del plan de modernización. En cada caso, el objetivo es analizar el signo y la magnitud de los efectos de las variables explicativas sobre la longitud del rango, considerando una población homogénea respecto a los factores sistemáticos que afectan a la variable aleatoria $T$ (Kiefer, 1988; Lancaster, 1990).

La longitud del intervalo de tiempo analizado comienza en el año en el cual aparece la tecnología en el mercado, dado que es el año en el cual las CC.RR. pasan a ser potenciales adoptantes. Algunas CC.RR. no han adoptado tecnología en el momento de la toma de datos y la finalización del intervalo se desconoce, aunque este puede ocurrir en el futuro. Para estos casos, el procedimiento estadístico es censurar por la derecha la duración del intervalo al final del periodo de observación, indicando que para esos casos el proceso continua, y por lo tanto, ha de tenerse en cuenta su naturaleza de censurado, siendo los mecanismos del evento y la censura estadísticamente independientes.

La probabilidad de transición a un nuevo estado está relacionada con la finalización del intervalo en $\mathrm{AD}$, por lo que éste se puede interpretar como la probabilidad de que una $\mathrm{CR}$ que no tiene tecnología de distribución y control del riego la adopte en un corto espacio de tiempo $d t$ después de $t$, condicionado a que ésta no la ha adoptado todavía. La probabilidad media de dejar este estado por periodo de tiempo unitario y por un corto intervalo de tiempo $d t$ después de $t$ vendría definida por la función de riesgo.

Formalmente, $f(t)$ es la función de densidad de probabilidad continua de la variable aleatoria $T$, donde $t$, es la finalización del rango. Su correspondiente función acumulada estará definida por:

$$
F(t)=\int_{0}^{t} f(s) d s=\operatorname{Pr}(T \leq t)
$$


Equivalentemente, la distribución de $T$ puede ser expresada por la función de supervivencia $S(t)$, que da la probabilidad de que la longitud del intervalo es al menos $t$, es decir, la probabilidad de que la variable aleatoria $T$ exceda a $t$.

$$
S(t)=1-F(t)=\operatorname{Pr}(T>t)
$$

La función de riesgo, denotada con $h(t)$, especifica la tasa instantánea de finalización del intervalo en $T=t$, condicionado a la supervivencia en el tiempo $t$, definida formalmente como:

$$
h(t)=\operatorname{Lim}_{d t \rightarrow 0} \frac{P(t \leq T<t+d t \mid T \geq t)}{d t}=\operatorname{Lim}_{d t \rightarrow 0} \frac{F(t+d t)-F(t)}{d t(1-F(t))}=\frac{f(t)}{S(t)}
$$

La función de riesgo puede ser interpretada como una versión de probabilidades condicionales continuas en el tiempo (en este caso, probabilidad condicional de adoptar), siendo $F(t), S(t)$ y $h(t)$ diferentes vías de expresión de la distribución de $T$. Para una CR, $1-S(t)$ proporcionará la probabilidad de que ésta adopte la innovación en el tiempo $t$, pero a nivel agregado, considerando todas aquellas CR activas, $1-S(t)$ representará la difusión esperada de la innovación entre los potenciales adoptante, es decir, la proporción de CR que ha adoptado tecnología en cada instante $t$.

El modelo de riesgo proporcional ajusta la función de riesgo instantáneo de sobrevivir a $t$, considerando los tiempos de supervivencia censurados. La función de riesgo está compuesta por una parte que refleja los efectos de las variables explicativas $(\lambda)$ y por la función de riesgo base $\left(h_{0}\right)$, que puede ser semiparamétrica, como en la regresión de Cox, o seguir una forma funcional concreta según el patrón $h_{0}(t)$ (Cleves et al., 2002; Kiefer, 1988). La función de riesgo presenta la siguiente ecuación general:

$$
h(t, X)=h_{0}(t) \lambda
$$

donde $\lambda \equiv \exp \left(\beta^{\prime}, X\right)$.

En el modelo de riesgo proporcional propuesto por Cox (1972) se estiman, por parcial verosimilitud, las relaciones entre la tasa de riesgo y las variables explicativas sin tener que asumir una forma funcional concreta de la función de riesgo base y donde las covariables modifican esta función en el tiempo. Los modelos semiparamétricos presentan la ventaja de no asumir una forma para la función de riesgo. Sin embargo, los modelos paramétricos son más eficientes en el uso de la información proporcionada por los datos porque, a diferencia de los métodos semiparamétricos, éstos no ignoran qué está sucediendo con las covariables en los periodos de tiempo donde no se producen adopciones. Por ello, la función de riesgo base puede ser parametrizada siguiendo diferentes formas funcionales como la exponencial, Weibull, Gompertz, etc. (Cleves et al., 2002; Kiefer, 1988). 
Usualmente, $\lambda$ tiene una especificación exponencial para evitar restricciones en los parámetros estimados, garantizando la no negatividad y realizando una estimación e inferencia sencilla. La heterogeneidad es incorporada en el modelo como una combinación lineal de las características de las CC.RR. (X) definidas como:

$$
\beta^{\prime} X=\beta_{0}+\beta_{1} X_{1}+\beta_{2} X_{2}+\beta_{3} X_{3}+\ldots+\beta_{k} X_{k}
$$

Donde $X_{k}$ son las variables observadas para cada CR y $\beta_{k}$ son los parámetros a estimar.

Derivando respecto a $X_{k}$, según esta especificación en forma logarítmica, se obtiene que el parámetro $\beta_{k}$ es la sensibilidad de la probabilidad condicional de adoptar, expresada como elasticidad (Kiefer, 1988):

$$
\partial \ln h\left(t, X, \beta, h_{0}\right) / \partial X_{k}=\partial \ln q(\beta, X) / \partial X_{k}=\beta_{k}
$$

La función de riesgo proporcional implica que cambios proporcionales en la tasa de riesgo originados por las variables explicativas no dependen de la duración. Esta función para las CC.RR. queda definida por:

$$
h\left(t, X, \beta, h_{0}\right)=h_{0}(t) \exp \left(\beta^{\prime}, X\right)
$$

Los coeficientes estimados proporcionan los valores de $\beta$ y su signo, y representan el impacto sobre la función de riesgo. Estos coeficientes deberán interpretarse como $\exp (\beta)$, donde valores iguales a 1 supondrían no impacto sobre la función de riesgo. Sin embargo, valores mayores (menores) de 1 indicarían un impacto positivo (negativo) sobre la función de riesgo, de ahí la negativa relación entre la variable y la longitud del tiempo de adopción.

Entre las especificaciones de la forma funcional de $T$, en los modelos de duración, destacan las distribuciones Weibull y Gompertz, que permiten variar el riesgo relativo a lo largo del proceso de duración, mientras que la distribución exponencial es caracterizada por una función de riesgo constante, $h(t)=\lambda$, donde el parámetro $\lambda>0$ implica que el paso del tiempo no influye sobre la tasa de riesgo. La distribución Weibull se caracteriza por la función de riesgo $h(t)=\lambda \alpha t^{\alpha-1}$, con $\lambda>0$ y $\alpha>0$. Si $\alpha$ $>1$ la función de riesgo se incrementará con el tiempo, por el contrario, si $\alpha<1$ esta función decrecerá con el tiempo, mientras que si $\alpha=1$ la función colapsará en una función exponencial. De la misma manera, la distribución Gompertz, $h(t)=\lambda \exp$ $(\gamma t)$, con $\lambda>0$ y $\gamma>0$ incrementará con el paso del tiempo, mientras que si $\gamma<0$ la función decrecerá con el tiempo, colapsando igualmente en una función exponencial cuando $\gamma=0$. El Cuadro 1 recoge las formas funcionales de las diferentes parametrizaciones. 
CUADRO1

Formas funcionales para los modelos Exponencial, Weibull y Gompertz

\begin{tabular}{lccc}
\hline & Exponencial & Weibull & Gompertz \\
\hline Densidad acumulada, $F(t)$ & $1-\exp (-\lambda t)$ & $1-\exp \left(-\lambda t^{\alpha}\right)$ & $1-\exp \{\lambda / \gamma[1-\exp (\gamma t)]\}$ \\
Supervivencia, $S(t)$ & $\exp (-\lambda t)$ & $\exp \left(-\lambda t^{\alpha}\right)$ & $\exp \{\lambda \gamma[1-\exp (\gamma t)]\}$ \\
Riesgo, $h(t)$ & $\lambda$ & $\lambda \alpha t^{\alpha-1}$ & $\lambda \exp (\gamma t)$ \\
\hline
\end{tabular}

Fuente: Elaboración propia a partir de Cleves et al. (2002) y Kiefer (1988).

\section{Datos y situación}

En la Región de Murcia, dentro de la Cuenca del Segura, se pueden encontrar diferentes tipos de regadíos dependiendo de las características de la zona, del origen del agua y de la infraestructura hidráulica empleada. Los cuatro tipos de regadíos más característicos son (CHS, 1997): a) regadíos tradicionales, legalizaciones y ampliaciones de las Vegas del Segura, $b$ ) regadíos creados con las aguas procedentes del Trasvase Tajo-Segura, $c$ ) regadíos que se abastecen con aguas subterráneas, y $d$ ) regadíos abastecidos con la reutilización de recursos.

Las CC.RR. de la Región, que gestionan el $80 \%$ de la demanda agrícola (1.571 $\mathrm{hm}^{3} / \mathrm{año}$ ) se encuentran diseminadas entre las diferentes tipologías de regadíos (CHS, 1997). Presentan, por término medio ${ }^{5}$, una superficie de 650 ha, 50 comuneros por CR y un caudal de $1 \mathrm{~m}^{3} / \mathrm{s}$.

Según el Plan Hidrológico de la Cuenca del Segura (PHCS), los regadíos están formados por 64 unidades de demanda agraria ${ }^{6}$, de las cuales 43 se encuentran dentro de la Región de Murcia. Dadas las similitudes agronómicas e hídricas de las diferentes unidades de demanda, éstas se pueden agrupar en 7 macro-unidades de demanda agraria que conforman las zonas regables de la Región.

Atendiendo al número de CC.RR. que componen las macro-unidades de demanda, y de forma proporcional a él, se seleccionaron 29 CC.RR. Y dentro de cada macro-unidad se encuestaron aquellas que representaban las diferentes tipologías regadíos. Para ello se consideró la técnica de riego dominante, el principal origen de los recursos hídricos utilizados, el tamaño de la zona regable, la antigüedad de la CR y las orientaciones productivas.

Los datos utilizados para el estudio han sido obtenidos de las entrevistas personales realizadas a los dirigentes de las CC.RR. seleccionadas. La muestra abarca 86.656 hectáreas, superficie que representa el 64,5\% de la superficie regable de la Región, afecta a más de 67.000 regantes y recoge todas las zonas regables con sus diferentes

${ }^{5}$ Datos facilitados por la Consejería de Agricultura y Agua de la Comunidad Autónoma de la Región de Murcia.

${ }^{6}$ Según el PHCS, una unidad de demanda agrícola es una unidad diferenciable de gestión, bien por su origen de recursos, por sus condiciones administrativas, por su similaridad hidrológica, o por consideraciones estrictamente territoriales (CHS, 1997). 
orígenes de agua. La información recabada es relativa a las características básicas de la CR (situación geográfica, histórica, y agrícola), a las características tecnológicas, al origen del agua y al tipo de gestión de ésta (Cuadro 2).

Los diferentes orígenes del agua utilizada para riego y la forma de gestionarla por las CC.RR. hacen que la Cuenca del Segura posea varias tipologías de regadíos, ya que en ella se utilizan caudales procedentes de las cuencas del Río Segura y sus afluentes (21\%), del Tajo a través del Acueducto Tajo-Segura (45\%), subterráneos (31\%), desalados $(3 \%)$ y depurados como complemento de los caudales de abastecimiento principales.

\section{CUADRO 2}

Estadísticas descriptivas de las principales características de las CC.RR. de la Región de Murcia $(\mathbf{n}=\mathbf{2 9})$

\begin{tabular}{lcccc}
\hline \multicolumn{1}{c}{ Variable } & Media & Desv. Estándar & Mínimo & Máximo \\
\hline Agua superficial $(0=$ no; $1=$ sí) & 0,21 & 0,41 & 0 & 1 \\
Agua del Trasvase T-S $(0=$ no; $1=$ sí) & 0,45 & 0,51 & 0 & 1 \\
Agua subterránea $(0=$ no; $1=$ sí) & 0,31 & 0,47 & 0 & 1 \\
Agua Desalada $(0=$ no; $1=$ sí) & 0,03 & 0,18 & 0 & 1 \\
Año de creación $($ excepto tres CC.RR. & 1980 & 14 & 1943 & 2003 \\
$\quad$ inmemoriales $\left.{ }^{7}\right)$ & 22,00 & 38,24 & 0 & 100 \\
Garantía de suministro $(\%)$ & 4.297 & 1.597 & 1.000 & 7.000 \\
Dotación teórica $\left(\mathrm{m}^{3} /\right.$ ha) & 0,17 & 0,38 & 0 & 1 \\
Pozo de apoyo $(0=$ no; $1=$ sí) & 0,86 & 0,35 & 0 & 1 \\
Sistema tarifario $(0=$ fijo; $1=$ variable) & 0,13 & 0,06 & 0,003 & 0,34 \\
Precio de agua $\left(€ / \mathrm{m}^{3}\right)$ & 0,52 & 0,51 & 0 & 1 \\
Tipo de gestión de riego $(0=$ turnos; $1=$ demanda) & 0,76 & 0,43 & 0 & 1 \\
Subvenciones $(0=$ no; $1=$ sí) & 0,31 & 0,47 & 0 & 1 \\
Adopción en año de sequía $(0=$ no; $1=$ sí) & & & \\
\hline
\end{tabular}

Fuente: Elaboración propia.

La garantía de suministro se define como «el compromiso para satisfacer con una cierta probabilidad la concesión otorgada, estableciéndose ésta con referencia a la demanda bruta anual y a la probabilidad de disponer de suficientes reservas anuales de recursos hídricos» (Losada, 1997) y es, por término medio, el 22\% de la dotación teórica $\left(\approx 4.300 \mathrm{~m}^{3} / \mathrm{ha}\right.$ ). Además ésta se encuentra determinada por la zona geográfica y el origen del agua. Solamente el 10\% de las CC.RR. encuestadas son capaces de garantizar el $100 \%$ de la dotación a sus regantes, utilizando agua procedente de acuíferos. El 66\% de las CC.RR. no garantizan agua alguna al comienzo del año hidrológico, mientras que las CC.RR. que utilizan agua procedente del Tajo, que dependen trimestralmente de la situación hidrológica de los embalses de la cuenca cedente, no

\footnotetext{
${ }^{7}$ Dos de las CC.RR. de la región de Murcia analizadas datan del siglo XVI y una se constituyó a mediados del siglo XIX.
} 
tienen ninguna garantía. Con lo que el origen del agua está condicionando la disponibilidad de la misma9 .

Los problemas de garantía tienen su origen en la procedencia del agua, ya que las CC.RR. con dotaciones de cuenca dependen del caudal del río y del estado de sus embalses para que sean efectivas, mientras que las CC.RR. que captan el agua del subsuelo se encuentran con la limitación de la sobreexplotación del acuífero al que estén ligadas y a los problemas técnicos de las infraestructuras de extracción. Por otro lado, las concesiones otorgadas con las aguas procedentes del Tajo no se encuentran formalizadas, y las transferencias entre cuencas deben ser aprobadas trimestralmente sin posibilidad de programación anual. Por lo tanto, la disponibilidad de agua del trasvase se encuentra siempre condicionada a los recursos existentes en el Tajo y a las decisiones de una comisión.

Por todo ello, el $17 \%$ de las CC.RR. optaron por la realización de un pozo que pueda complementar los principales caudales utilizados por la $\mathrm{CR}$, independientemente de su origen. El uso de agua subterránea implica que la CR posee una fuente de agua con menor fluctuación intra-anual que la superficial y más independiente en cuanto a las decisiones políticas que hay en torno a las regulaciones de los recursos de la cuenca y trasvases.

Respecto al análisis técnico-económico, se puede ver que el alto grado de desarrollo hidráulico existente en la Región de Murcia permite conocer el consumo de agua que realiza cada agricultor, que unido a la madurez en la economía del agua presente, ha llevado a la mayoría de CC.RR. (86\%) a establecer un sistema tarifario variable en función del consumo que realiza cada agricultor. En casos excepcionales, se siguen respetando los Usos y Costumbres de la Huerta (Pérez Crespo, 1989), manteniendo un sistema tarifario fijo donde se paga una cantidad anual por hectárea. La escasez ha derivado en elevados controles de consumo y precios del agua $\left(0,13 € / \mathrm{m}^{3}\right)$.

El sistema de tarifas, y reparto de agua de la CR, dependerá en buena medida de la tecnología que posea para realizar una asignación de recursos eficiente, ya que no existe un mercado establecido en la zona para este recurso, y el reparto se realiza de forma proporcional a la superficie, siguiendo el criterio que Sprumont (1991) llamó regla uniforme, con la particularidad de que la dotación de agua asignada a los regantes se encuentra generalmente por debajo de su asignación ideal. Esto implicará que un sistema tarifario en función del consumo, más eficiente e incentivador del ahorro, requerirá de un sistema de organización y control del uso del agua apropiado. La implantación de este sistema de tarifas variable ha de facilitar la medición fiable de los caudales y consumos, y ha de ser aceptada por todos los comuneros. Esto permitirá a la CR facturar a cada agricultor la cantidad de agua utilizada, así como, tener el control de la situación hídrica de la comunidad.

El papel de las CC.RR. como instituciones, a la hora de organizar y controlar el reparto del agua entre sus comuneros, se basa en la organización del riego y el control de los consumos. El sistema de distribución se realiza desde dos perspectivas diferentes: el riego a la demanda, dentro de la asignación de cada regante (52\%), o el riego

\footnotetext{
${ }^{8}$ Para más detalles de los regadíos de la región de Murcia y las características de las CC.RR., ver (Alcón et al., 2004).
} 
por turnos (48\%), donde se suelen establecer tandas de cabeza a cola del eje principal de distribución. Estos sistemas, están muy influenciados por la infraestructura de distribución utilizada, ya que, cuando se dispone de un sistema de distribución moderno con tuberías, contadores, válvulas de control, automatismos, etc., se favorece la distribución y racionalización del uso del agua.

El 95\% de las CC.RR. que han modernizado sus instalaciones se han acogido a las ayudas institucionales promovidas por el PNR. Nueve de ellas han adoptado durante el periodo de sequía de los años 2004-2005.

La variable explicada es el rango de tiempo comprendido entre el año en el que la CR se constituye, o el año 1975 si es que esta lo hizo con anterioridad, y el año en el que comienza la ejecución de un plan de modernización de regadío basado en la instalación de tecnología que permita regular la distribución y controlar los caudales utilizados. En algunos casos, la CR no tiene previsto, ni realizado, plan de modernización alguno, y la posible fecha futura de adopción se desconoce. Estos casos serán censurados al último año del periodo analizado considerándose su naturaleza al estimarse.

El Gráfico 1 expone el número de CC.RR. adoptantes de la muestra durante el periodo analizado. Aunque esta tecnología se encontraba ya en el mercado, no fue hasta el año 1988 cuando la primera CR tomó la decisión de implantarla en sus tierras de cultivo. De las 29 CC.RR. analizadas, seis han sido censuradas en el año 2005, ya que en esa fecha no habían adquirido tecnología.

\section{GRÁFICO 1}

Número de CC.RR. que adoptan tecnología en la muestra

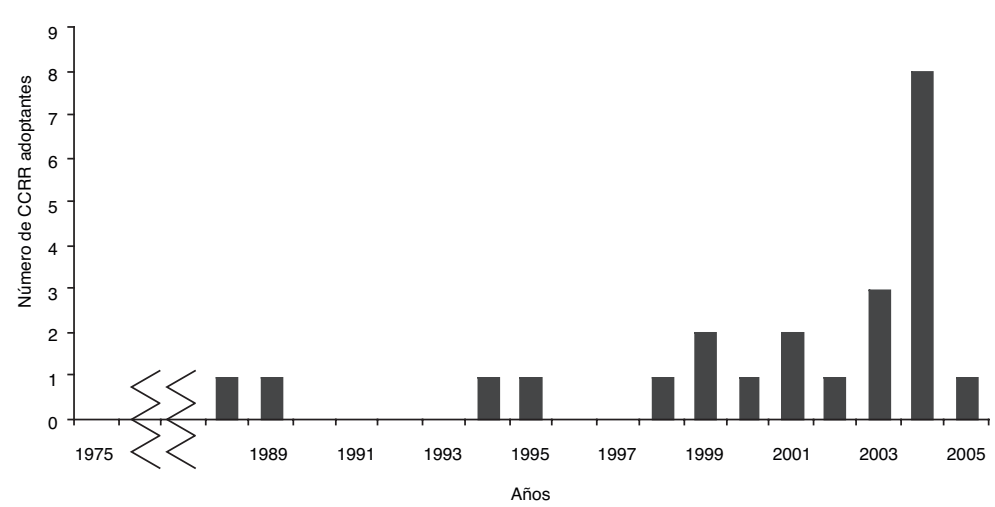

Fuente: Elaboración propia.

\section{Resultados}

$\mathrm{Al}$ analizar la proporción de CC.RR. que adoptan tecnología de riego, respecto al número de CC.RR. constituidas en esa fecha para el periodo 1975-2005, y en base a la ejecución de un plan de modernización de regadíos, que mejore la infraestructura 
de distribución del agua de riego e incremente la eficiencia técnica de su uso, se aprecian tres fases claramente diferenciadas.

La primera fase del período analizado comprende desde 1975 hasta la entrada de España en la Comunidad Económica Europea en 1986, período en el que las CC.RR. de la Región no realizaron inversiones en tecnología. En la segunda fase, las inversiones en materia de modernización de regadíos no llegaban al 30\% de las CC.RR. analizadas. En esta fase, que va desde el año 1986 hasta el 2000, concluyeron los estudios de base sobre los regadíos nacionales, se adecuaron las previsiones de actuación en materia de regadíos a las políticas comunitarias y entró en vigor el PNR. A pesar de que la tecnología se encontraba en el mercado durante la tercera fase (20012005), ha sido en los últimos 5 años cuando se ha producido el despegue tecnológico que abarca casi al $80 \%$ de la muestra.

Para analizar el conjunto de los tiempos de supervivencia de la muestra, la función de supervivencia, estimada según Kaplan-Meier, es la más utilizada. Este acercamiento no paramétrico no asume ninguna distribución subyacente de los tiempos de supervivencia y solamente es identificada cuando la adopción ocurre. El Gráfico 2 muestra la función de supervivencia estimada para el conjunto de CC.RR. Esta función indica, para cada periodo, la probabilidad que tiene una CR de no adoptar tecnología, es decir, el tiempo de retraso que sufre la CR desde que se constituye hasta que adopta. La duración del periodo de adopción tiene un valor medio de 19,5 años y sus variaciones son inversamente proporcionales a la supervivencia en el estado intransitivo.

\section{GRÁFICO 2}

Estimación no paramétrica de la función de supervivencia de las CC.RR. de la región de Murcia (Estimador Kaplan-Meier)

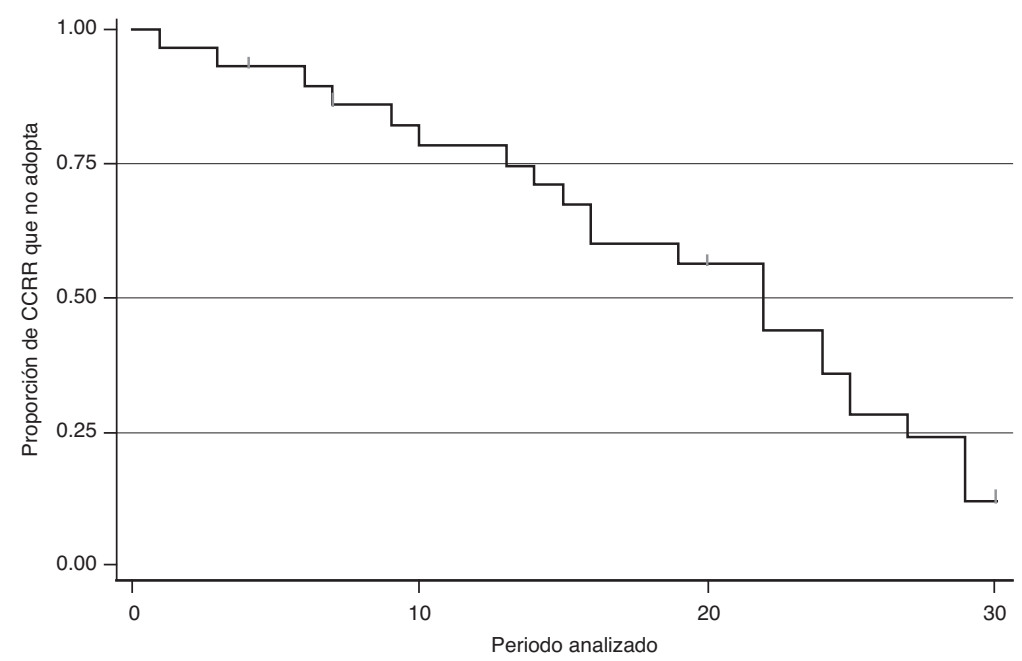

Fuente: Elaboración propia. 
Para considerar los efectos de las covariables, que explican el retraso en el tiempo de adopción utilizado por cada $\mathrm{CR}$, se han realizado seis modelos alternativos que estiman los parámetros de la ecuación de riesgo, y la dirección de su efecto. Las estimaciones se han realizado utilizando parametrizaciones Weibull y Gompertz que recogen la influencia temporal y permiten acomodar la distribución exponencial. También se ha considerado la censura de los datos en la estimación de la función de verosimilitud.

Las variables utilizadas en los modelos reflejan las principales características de disponibilidad, institucionales y económicas de las CC.RR. Estas variables son previas a la decisión de implantar un plan de modernización de regadíos. Las variables, recogidas por encuesta, que representan el nivel tecnológico de las CC.RR. han sido excluidas del análisis por representar características intrínsecas a la modernización. Además, las variables correlacionadas con las seleccionadas y las que presentan problemas de multicolinealidad han sido excluidas del análisis.

El Cuadro 3 expone los resultados de los seis modelos, donde los parámetros expresan los coeficientes estimados para las diferentes formas funcionales que pueden afectar sobre la velocidad de difusión. El valor del estadístico $t$ de cada variable respecto a la hipótesis nula de no impacto sobre la función de riesgo, $\beta=0$, viene expresado entre paréntesis y el signo de los coeficientes indica la dirección del impacto de la variable sobre la tasa de adopción esperada.

Los modelos 1 y 2 utilizan las mismas variables pero diferentes formas funcionales. Para poder compararlos se ha utilizado el test de Vuong (Vuong, 1989) y el criterio de información de Akaike (AIC) (Akaike, 1974). Ambos indicadores permiten afirmar la superioridad del modelo Gompertz para recoger la estructura de los datos. De igual manera, los modelos 3 y 4 representan formas funcionales distintas y se encuentran anidados en los modelos 1 y 2 respectivamente. Atendiendo a los mismos criterios, el modelo 4 mejora la forma funcional del modelo 3. El modelo 4 es el más restringido de todos los que presentan una forma funcional Gompertz y se encuentra anidado en los modelos 2, 5 y 6 . La prueba de razón de verosimilitud (LR) entre los modelos alternativos $(z=2,5,6)$ rechazó que las variables incluidas mejoraran significativamente las estimaciones del modelo 4 . Atendiendo a estas consideraciones y al análisis gráfico de los residuos (Cox y Snell, 1968), se puede afirmar que el modelo 4 es el que mejor ajuste presenta.

El modelo 4 presenta una parametrización Gompertz con un coeficiente estimado $(\gamma)$ mayor que cero. Esto sugiriere una dependencia positiva de la duración, es decir, la probabilidad condicional de adoptar incrementa con el paso del tiempo, reduciendo el tiempo esperado de adopción.

Los coeficientes del modelo de riesgo proporcional han de interpretarse de forma exponencial (Gráfico 3). Los riesgos relativos representan el impacto marginal de las características de las CC.RR. sobre la velocidad de difusión. Valores de las tasas de riesgo relativo expresadas en términos exponenciales mayores de la unidad indican impactos positivos sobre la probabilidad de adoptar, y menores de uno, negativos, de ahí la negativa relación entre el impacto de la variable y la longitud del tiempo de adopción. Por ejemplo, la tasa de riesgo de la variable Pozo de apoyo, en el modelo 4 es de 3,8 , indicando que las CC.RR. que poseen este tipo de pozos incrementan de 
CUADRO 3

Estimación de los modelos de riesgo proporcional para el tiempo de adopción en las CC.RR.

\begin{tabular}{|c|c|c|c|c|c|c|}
\hline \multirow{2}{*}{ Variables } & Modelo 1 & Modelo 2 & Modelo 3 & Modelo 4 & Modelo 5 & Modelo 6 \\
\hline & Weibull & Gompertz & Weibull & Gompertz & Gompertz & Gompertz \\
\hline Dotación teórica & $\begin{array}{c}0,00029 \\
(1,59)\end{array}$ & $\begin{array}{c}0,00036 \\
(1,79)\end{array}$ & & & & $\begin{array}{c}0,0034 \\
(1,75)\end{array}$ \\
\hline Pozo de apoyo & $\begin{array}{c}1,27 \\
(2,13)\end{array}$ & $\begin{array}{c}1,75 \\
(2,71)\end{array}$ & $\begin{array}{c}0,94 \\
(1,76)\end{array}$ & $\begin{array}{c}1,33 \\
(2,34)\end{array}$ & $\begin{array}{c}1,05 \\
(1,62)\end{array}$ & $\begin{array}{c}1,39 \\
(0,91)\end{array}$ \\
\hline Sistema tarifario & $\begin{array}{c}2,06 \\
(1,72)\end{array}$ & $\begin{array}{c}2,51 \\
(2,08)\end{array}$ & $\begin{array}{c}1,41 \\
(1,29)\end{array}$ & $\begin{array}{c}1,66 \\
(1,54)\end{array}$ & $\begin{array}{c}0,87 \\
(0,74)\end{array}$ & $\begin{array}{c}1,83 \\
(1,46)\end{array}$ \\
\hline Precio de agua & & & & & $\begin{array}{c}8,89 \\
(1,78)\end{array}$ & $\begin{array}{l}10,99 \\
(2,04)\end{array}$ \\
\hline Tipo de gestión de riego & $\begin{array}{c}-0,24 \\
(-0,42)\end{array}$ & $\begin{array}{c}-0,32 \\
(-0,57)\end{array}$ & & & & $\begin{array}{c}-0,82 \\
(-1,41)\end{array}$ \\
\hline Subvenciones & $\begin{array}{c}1,55 \\
(1,34)\end{array}$ & $\begin{array}{c}2,06 \\
(1,76)\end{array}$ & $\begin{array}{c}1,76 \\
(1,62)\end{array}$ & $\begin{array}{c}2,23 \\
(2,04)\end{array}$ & $\begin{array}{c}2,27 \\
(2,00)\end{array}$ & $\begin{array}{c}2,44 \\
(2,10)\end{array}$ \\
\hline Adopción en año de sequía & $\begin{array}{l}-1,31 \\
(-2,11)\end{array}$ & $\begin{array}{c}-1,88 \\
(-2,72)\end{array}$ & $\begin{array}{c}-0,82 \\
(-1,81)\end{array}$ & $\begin{array}{c}-1,19 \\
(-2,45)\end{array}$ & $\begin{array}{c}-1,32 \\
(-2,62)\end{array}$ & $\begin{array}{c}-2,24 \\
(-3,14)\end{array}$ \\
\hline Constante & $\begin{array}{c}-11,92 \\
(5,11)\end{array}$ & $\begin{array}{c}-10,99 \\
(5,36)\end{array}$ & $\begin{array}{l}-10,10 \\
(-5,15)\end{array}$ & $\begin{array}{c}-8,93 \\
(-5,57)\end{array}$ & $\begin{array}{c}-9,51 \\
(-5,87)\end{array}$ & $\begin{array}{l}-11,94 \\
(-5,54)\end{array}$ \\
\hline Parámetros de $h_{0}$ & $\begin{array}{c}\alpha=2,54 \\
(5,45)\end{array}$ & $\begin{array}{c}\gamma=0,18 \\
(4,71)\end{array}$ & $\begin{array}{c}\alpha=2,40 \\
(4,73)\end{array}$ & $\begin{array}{c}\gamma=0,17 \\
(4,53)\end{array}$ & $\begin{array}{c}\gamma=0,18 \\
(4,61)\end{array}$ & $\begin{array}{c}\gamma=0,21 \\
(4,74)\end{array}$ \\
\hline Log-likelihood & $-19,63$ & $-14,24$ & $-20,96$ & $-15,98$ & $-14,58$ & $-12,45$ \\
\hline LR Chi-cuadrado & 17,89 & 24,60 & 15,22 & 21,12 & 23,93 & 28,18 \\
\hline Prob $>$ Chi-cuadrado & 0,0065 & 0,0004 & 0,0043 & 0,0003 & 0,0002 & 0,0002 \\
\hline Vuong test $_{(\mathrm{W}-\mathrm{G})}$ & & $-4,73$ & & $-13,78$ & & \\
\hline AIC & 55,25 & 44,49 & 53,93 & 43,96 & & \\
\hline $\mathrm{LR}=-2\left(L L_{4}-L L_{z}\right)$ & & $\begin{array}{c}3,48 \\
\chi_{0,05,2}^{2}=5,99\end{array}$ & & & $\begin{array}{c}2,8 \\
\chi_{0,05,1}^{2}=3,84\end{array}$ & $\begin{array}{c}7,06 \\
\chi_{0,05,3}^{2}=7,82\end{array}$ \\
\hline
\end{tabular}

Fuente: Elaboración propia. El valor entre paréntesis corresponde con el valor t-Student.

forma proporcional la función de riesgo en casi cuatro veces a sus homólogas sin pozo, manteniendo el resto de variables constantes.

Como ya se ha indicado, las CC.RR. que poseen pozo, para la extracción de agua subterránea, tienen una tasa instantánea de adopción superior a las CC.RR. que no lo tienen. El poseerlo supone un incremento de la velocidad de difusión de un plan de modernización de regadíos. Tal y como apuntaron, Green et al. (1996), Schuck y Green (2001) y Green y Sunding (1997) la posibilidad de acceder a una fuente alternativa de suministro de agua incrementa la probabilidad de adoptar tecnología de riego. No obstante, los trabajos de Negri y Brooks (1990) y Carey y Zilberman (2002) comprobaron que las fuentes alternativas de suministro de agua, medidas 
como el acceso al uso de agua superficial en una zona donde predominaba el uso de agua subterránea o la existencia de un mercado de agua, afectaba de forma negativa a la adopción de sistemas de riego.

Igualmente, las CC.RR. que utilizan un sistema tarifario variable y discriminante, en función del consumo, incrementarán la velocidad de difusión, reduciendo más de cinco veces el tiempo esperado de adopción. Así, las CC.RR. que poseen unos sistemas tarifarios más eficientes conseguirán una adopción más temprana, dado que, generalmente, mayores tasas de eficiencia conducen a mayores tasas de adopción (Antle y Crissman, 1990; Ghosh et al., 1994).

Just y Zilberman (1983) expusieron que el empleo de políticas que subvencionaran factores de producción, reduciendo riesgos dentro del proceso de difusión, alentaría la adopción de nuevas tecnologías, tal y como recoge la magnitud y el signo de la variable Subvenciones.

A pesar de que los periodos de escasez han favorecido la adopción de tecnologías ahorradoras de recursos (Carey y Zilberman, 2002; Foltz, 2003), el modelo sugiere que las CC.RR. que no adoptaron en un año de sequía tardaron menos en hacerlo. Por ello, uno de los factores que afectaron a la adopción de tecnología de distribución y control del agua de riego por las CC.RR. de la Región de Murcia fue la garantía en el suministro del agua. Como la situación de escasez de la zona estudiada es tan acusada, el adoptar en un año de sequía reduce de forma considerable el tiempo esperado de adopción, dado que las dotaciones asignadas en estos períodos apenas si alcanzan para la supervivencia de las plantaciones. La variable Sequía sugiere que si la CR no dispone de agua suficiente, retrasará la decisión de adoptar a períodos que garanticen el funcionamiento y amortización de las inversiones.

El Gráfico 3 representa la probabilidad condicional que tiene una CR de adoptar mejores tecnologías de distribución y control de agua en los diferentes periodos de tiempo, con respecto a los valores medios de las variables explicativas recogidas en el modelo 4. Además, se puede ver cómo la probabilidad condicional de adoptar crece considerablemente con el tiempo, especialmente a partir del año 21 (1996) cuando se supera el 10\%, ya que a partir de este momento la probabilidad condicional se triplica en la mitad de tiempo. Por término medio, la probabilidad de que una CR de la zona analizada, constituida en el año 1975 o anterior, que no posee tecnología de distribución y control del riego en el año 1984, adopte en el año 1985, es del 1,5\%, condicionado a que ésta no la haya adoptado aún. Pero si se analiza esta probabilidad en el año 2000, el valor medio de dejar el estado de no adoptante se incrementará al $20 \%$, siendo la probabilidad condicional de adoptar esta tecnología al final del período analizado superior al $44 \%$.

Si atendemos al tiempo de supervivencia estimado por el modelo cuatro (Gráfico 4), la probabilidad de que una CR no haya adoptado en cada período de tiempo $t$ es muy elevada en el primer tercio del periodo analizado, habiendo adoptado a los diez años de constituirse solamente el 7,1\% de las CC.RR. En los últimos años, el porcentaje de CC.RR. que no han adoptado tecnología decrece considerablemente, alcanzándose en el año 2000 una probabilidad de sobrevivir a la adopción del 33,3\%. Estas probabilidades estarán igualmente muy condicionadas a los efectos de las variables explicativas del modelo. 


\section{GRÁFICO 3}

Función de riesgo proporcional para la adopción de tecnología en las CC.RR.

(Distribución Gompertz) (1975-2005)

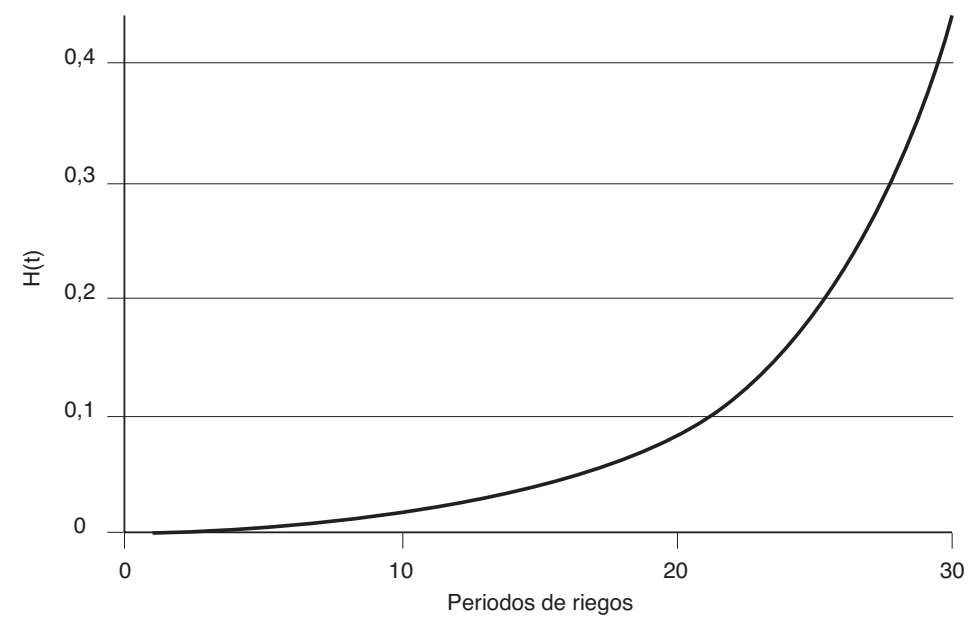

Fuente: Elaboración propia.

\section{GRÁFICO 4}

Función de supervivencia en la adopción de tecnología de las CC.RR.

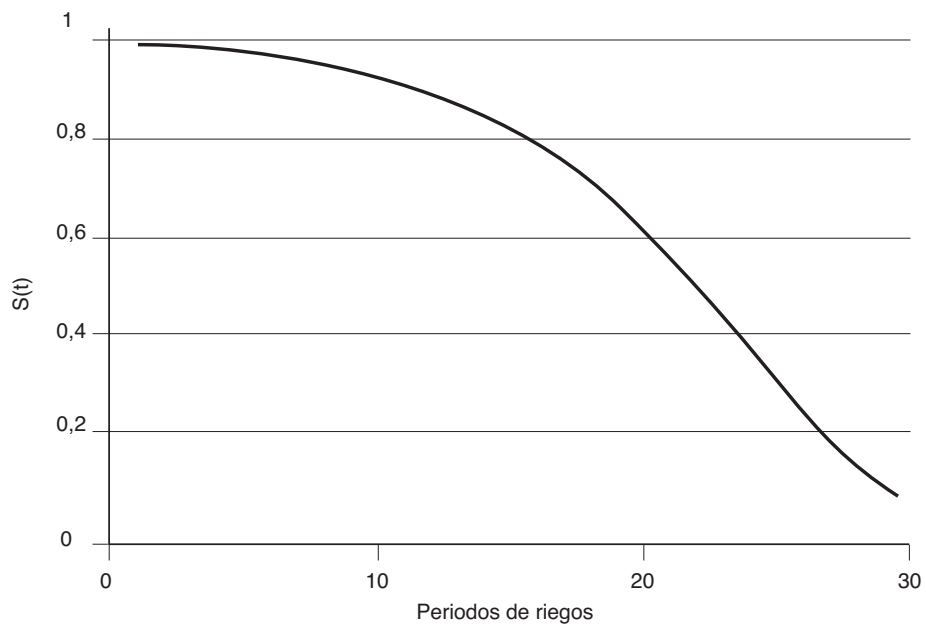

Fuente: Elaboración propia. 


\section{Conclusiones}

El estudio ha analizado la dinámica de la adopción de tecnología de distribución y control del agua de riego que mejora la productividad del agua entre las CC.RR. en la Región de Murcia.

El AD estima el retraso en la adopción de las CC.RR. asociado con sus características, superando las deficiencias de los modelos de adopción dicotómicos tradicionalmente utilizados. Los resultados sugieren que la probabilidad de adoptar tecnología de riego depende positivamente del paso del tiempo, y que la variable que establece una garantía de suministro, a través de una fuente alternativa, estimula la decisión de adoptar.

Por otro lado, el uso de un sistema de tarifas dependiente del consumo individual de cada agricultor, que incentive el ahorro y consiga una asignación más eficiente de los escasos recursos hídricos de la zona, fomenta el uso de modernas tecnologías de riego.

Asimismo, los períodos de sequía han afectado de forma negativa a la adopción, dado que los agricultores, y por consecuente las CC.RR., necesitan de un suministro de agua mínimo que garantice su decisión de adoptar.

Los efectos de las políticas de subvenciones planteadas por el PNR han quedado manifiestos en el trabajo de adopción, afectando de forma positiva a la probabilidad de ejecutar un plan de modernización de regadíos que incremente la eficiencia en el uso del agua.

La adopción de tecnología de distribución y control del agua permitirá a las CC.RR. disponer de los dispositivos de medición y gestión requeridos para la implementación de la DMA. En este sentido, se debería prestar atención no solo a las variables identificadas en este trabajo, sino a todas aquellas políticas que mejoren las aplicaciones de las directrices de la DMA generando un efecto multiplicador en la eficiencia de la gestión de las CC.RR., como la adopción de tecnologías ahorradoras de agua, las políticas tarifarias y de revisión de concesiones y la implantación de mercados de agua.

Tal y como se desprende de la «Paradoja de Jevons», en algunas ocasiones, una mejora de la eficiencia técnica del uso del agua no conduce a su ahorro, sino que es probable que estimule el consumo del recurso de forma contraria a aquello para lo cual fue pensado (Alcott, 2005). Según este fenómeno, las políticas de eficiencia serían contraproducentes y deberían compensarse con aumentos del precio del agua o reducciones de las dotaciones. Y aunque ésto podría inducir a pensar sobre la existencia de esta controversia en la Región de Murcia, la escasez de recursos ha provocado que se incrementen los precios del agua y se limiten los perímetros de las zonas regables. Por lo que el problema planteado es el reparto de los recursos disponibles de forma proporcional a la superficie de cada regante, que en la mayoría de los años, se encuentran por debajo de las dotaciones teóricas recogidas por el PHCS.

Por lo tanto, la adopción de tecnología por las CC.RR., de igual modo que la gestión hídrica que éstas realizan, mejorará el uso de los recursos naturales de la Región de Murcia, contribuyendo al mantenimiento de la actividad agraria y la sostenibilidad medioambiental de la Región. 


\section{Bibliografía}

Abdulai, A. y Huffman, W.E. (2005). «The diffusion of new agricultural technologies: The case of crossbred-cow technology in Tanzania». American Journal of Agricultural Economics, 87:645-659.

Adesina, A.A. y Baiduforson, J. (1995). «Farmers' perceptions and adoption of new agricultural technology: Evidence from analysis in Burkina Faso and Guinea, West Africa». Agricultural Economics, 13:1-9.

Adesina, A.A. y Zinnah, M.M. (1993). «Technology characteristics, farmers’ perceptions and adoption decisions: A Tobit model application in Sierra Leone». Agricultural Economics, 9:297-311.

Akaike, H. (1974). «A new look at the statistical model identification». IEEE Transactions on Automatic Control, 19:716-723.

Alcón, F., de Miguel, M.D. y Fernández-Zamudio, M.A. (2006). «Modelización de la difusión de la tecnología de riego localizado en el Campo de Cartagena». Revista Española de Estudios Agrosociales y Pesqueros, 210:227-245.

Alcón, F., de Miguel, M.D. y Smith, H.B. (2004). «El agua en la agricultura de la Región de Murcia». V Congreso de la Asociación Española de Economía Agraria. Santiago de Compostela.

Alcott, B. (2005). «Jevons’ paradox». Ecological Economics, 54:9-21.

Antle, J.M., y Crissman, C.C. (1990). «Risk, efficiency, and the adoption of modern crop varieties: Evidence from the Philippines». Economic Development and Cultural Change, 38:517-537.

Burton, M., Rigby, D. y Young, T. (2003). «Modelling the adoption of organic horticultural technology in the UK using duration analysis». Australian Journal of Agricultural \& Resource Economics, 47:29-54.

Box, M. (1992). «El regadío medieval en España: época árabe y conquista romana». En Gil, A. y Morales, A. (Eds.): Hitos históricos de los regadios españoles. MAPA, Madrid, pp. 49-90.

Carey, J.M. y Zilberman, D. (2002). «A model of investment under uncertainty: Modern irrigation technology and emerging markets in water». American Journal of Agricultural Economics, 84(1):171-183.

Cason, T.N. y Uhlaner, R.T. (1991). «Agricultural productions impact on water and energy demand: A choice modeling approach». Resources and Energy, 13:307-321.

Caswell, M., Lichtenberg, E. y Zilberman, D. (1990). «The effects of pricing policies on water conservation and drainage». American Journal of Agricultural Economics, 72:883-890.

Caswell, M. y Zilberman, D. (1985). «The choices of irrigation technologies in California». American Journal of Agricultural Economics, 67:223-234.

CHS (1997). Plan hidrológico de la Cuenca del Segura. Confederación Hidrográfica del Segura. http://www.chsegura.es/chs/.

Cleves, M.A., Gould, W.W y Gutiérrez, R.G. (2002). An introduction to survival analysis using Stata. Stata Corporation, College Station, Texas.

Cox, D.R. (1972). «Regression models and life-tables». Journal of the Royal Statistical Society. Series B (Methodological), 34:187-220.

Cox, D.R. y Snell, E.J. (1968). «A general definition of residuals (with discussion)». Journal of the Royal Statistical Society. Series B (Methodological), 39:248-275.

Dinar, A. y Yaron, D. (1992). «Adoption and abandonment of irrigation technologies». Agricultural Economics, 6:315-332. 
D’Emden, F.H., Llewellyn, R.S. y Burton, M.P. (2006). «Adoption of conservation tillage in Australian cropping regions: An application of duration analysis». Technological Forecasting and Social Change, 73:630-647.

Feder, G. (1980). «Farm size, risk aversion and the adoption of new technology under uncertainty». Oxford Economic Papers, 32:263-283.

Feder, G. y Umali, D.L. (1993). «The adoption of agricultural innovations: A review». Technological Forecasting and Social Change, 43:215-239.

Foltz, J.D. (2003). «The economics of water-conserving technology adoption in Tunisia: An empirical estimation of farmer technology choice». Economic Development and Cultural Change, 51:359-373.

Fuglie, K.O. y Kascak, C.A. (2001). «Adoption and diffusion of natural-resource-conserving agricultural technology». Review of Agricultural Economics, 23:386-403.

Gatignon, H. y Robertson, T.S. (1991). «Innovative decision processes». En Robertson, T.S. y Kassarjian, H.H. (Eds.), Handbook of Consumer Behaviour. Prentice-Hall, Englewood Cliffs, NJ., pp. 316-348.

Ghosh, S., McGuckin, J.T. y Kumbhakar, S.C. (1994). «Technical efficiency, risk attitude, and adoption of new technology: The case of the U.S. dairy industry». Technological Forecasting and Social Change, 46:269-278.

Green, G. y Sunding, D. (1997). «Land allocation, soil quality, and the demand for irrigation technology». Journal of Agricultural and Resource Economics, 22:367-375.

Green, G., Sunding, D., Zilberman, D. y Parker, D. (1996). «Explaining irrigation technology choices: A microparameter approach». American Journal of Agricultural Economics, 78:1064-1072.

Hayami, Y. y Ruttan, V.W. (1985). Agricultural development: An international perspective. Johns Hopkins University Press, Baltimore.

Just, R.E. y Zilberman, D. (1983). «Stochastic structure, farm size and technology adoption in developing countries». Oxford Economic Papers, 35:307-328.

Kiefer, N.M. (1988). «Economic duration data and hazard functions». Journal of Economic Literature, 26:646-679.

Lancaster, T. (1990). The econometric analysis of transition data. Cambridge University Press, Cambridge.

Lindner, R. (1987). «Adoption and diffusion of technology: An overview». En Champ, B.R., Highly, E. y Remenyi, J.V. (Eds.): Technological Change in porharvest Handling and Transportation of Grain in the Humid Tropic. Australian Centre for International Agricultural Research, Bangkok, Tailandia, pp. 144-151.

Losada, A. (1997). «Glosario sobre sistemas de riego». Ingeniería del Agua, 4:55-68.

MAPA (2001). Plan nacional de regadios, horizonte-2008. Ministerio de Agricultura Pesca y Alimentación, Dirección General de Desarrollo Rural, Madrid.

Marra, M., Pannell, D.J. y Abadi-Ghadim, A.K. (2003). «The economics of risk, uncertainty and learning in the adoption of new agricultural technologies: Where are we on the learning curve?». Agricultural Systems, 75:215-234.

MMA (2007). Precios y costes de los servicios del agua en España. Ministerio de Medio Ambiente, Madrid.

Nadal, E. (1980). «Los orígenes del regadío en España». Revista de Estudios Agro-Sociales, 113:7-37.

Negri, D. y Brooks, D. (1990). «Determinants of irrigation technology choice». Western Journal of Agricultural Economics, 15:213-223. 
Pérez Crespo, A. (1989). Usos y costumbres en la aparecería de la provincia de Murcia. Universidad de Murcia, Murcia.

Rogers, E.M. (2003). Diffusion of innovations. Free Press, New York.

Schaible, G., Kim, C.S. y Whittlesey, N. (1991). «Water conservation potential from irrigation technology transitions in the Pacific-Northwest». American Journal of Agricultural Economics, 16:194-206.

Schuck, E.C. y Green, G. (2001). «Field attributes, water pricing, and irrigation technology adoption». Journal of Soil and Water Conservation, 56:293-298.

Sidibe, A. (2005). «Farm-level adoption of soil and water conservation techniques in northern Burkina Faso». Agricultural Water Management, 71:211-224.

Smale, M., Just, R.E. y Leathers, H.D. (1994). «Land allocation in HYV adoption models: An investigation of alternative explanations». American Journal of Agricultural Economics, 76:535-546.

Souza Filho, H.M., Young, T. y Burton, M.P. (1999). «Factors influencing the adoption of sustainable agricultural technologies: Evidence from the State of Espirito Santo, Brazil». Technological Forecasting and Social Change, 60:97-112.

Sprumont, Y. (1991). «The division problem with single-peaked preferences: A characterization of the uniform allocation rules». Econometrica, 59:509-519.

Stoneman, P. (1986). «Technological diffusion: The viewpoint of Economic Theory». Richerche Economiche, 40:585-606.

Sumpsi, J. M., Garrido, A., Blanco, M., Varela, C. e Iglesias, E. (1998). Economía y política de gestión del agua en la agricultura. Ministerio de Agricultura, Pesca y Alimentación, Ed. Mundi-Prensa, Madrid.

Vuong, Q. (1989). «Likelihood ratio tests for model selection and nonnested hypotheses». Econometrica, 57:307-333.

Zilberman, D., Dinar, A., MacDougall, N., Khanna, M., Brown, C. y Castillo, F. (1995). Individual and institutional responses to drought: The case of California agriculture. Working Paper, Dept. of Agr. and Res. Econ., University of California, Berkeley.

Wulfhorst, J.D. (2002). «Irrigation, community, and historical development along the upper Snake River». Agricultural History, 76:434-447. 